\title{
On starlikeness of certain integral transforms
}

\author{
by S. Ponnusamy (Kanpur)
}

Abstract. Let $A$ denote the class of normalized analytic functions in the unit disc $U=\{z:|z|<1\}$. The author obtains fixed values of $\delta$ and $\varrho(\delta \approx 0.308390864 \ldots$, $\varrho \approx 0.0903572 \ldots)$ such that the integral transforms $F$ and $G$ defined by

$$
F(z)=\int_{0}^{z}(f(t) / t) d t \text { and } G(z)=(2 / z) \int_{0}^{z} g(t) d t
$$

are starlike (univalent) in $U$, whenever $f \in A$ and $g \in A$ satisfy $\operatorname{Re} f^{\prime}(z)>-\delta$ and $\operatorname{Re} g^{\prime}(z)>-\varrho$ respectively in $U$.

1. Introduction. Let $A$ denote the class of analytic functions $f$ defined in the unit disc $U=\{z:|z|<1\}$ and normalized so that $f(0)=f^{\prime}(0)-1=$ 0 . Let $S^{*}$ be the usual class of starlike (univalent) functions in $U$, i.e.

$$
S^{*}=\left\{f \in A: \operatorname{Re}\left[z f^{\prime}(z) / f(z)\right]>0, z \in U\right\}
$$

and let $R(\beta)=\left\{f \in A: \operatorname{Re} f^{\prime}(z)>\beta, z \in U\right\}, R(0) \equiv R(\beta<1)$.

In a recent paper [8], Singh and Singh proved that if $f \in R$, then $F \in S^{*}$, where

$$
F(z)=\int_{0}^{z}(f(t) / t) d t
$$

and in [3], Mocanu considered the Libera transform $G$ defined by

$$
G(z)=(2 / z) \int_{0}^{z} g(t) d t
$$

and showed that $g \in R$ implies $G \in S^{*}$.

In this note, we improve both the above results by showing that the same conclusions hold under a much weaker condition on $f$ and $g$ respectively.

1991 Mathematics Subject Classification: Primary 30C80; Secondary 30C25.

Key words and phrases: subordination, convex function, starlike function, univalent function. 
2. Preliminaries. We need the following lemma to prove our results.

Lemma A. Let $\Omega$ be a set in the complex plane $\mathbb{C}$. Suppose that the function $m: \mathbb{C}^{2} \times U \rightarrow \mathbb{C}$ satisfies the condition

$$
m(i x, y ; z) \notin \Omega
$$

for all real $x, y \leq-\left(1+x^{2}\right) / 2$ and all $z \in U$. If the function $p$ is analytic in $U$ with $p(0)=1$ and if $m\left(p(z), z p^{\prime}(z) ; z\right) \in \Omega, z \in U$, then $\operatorname{Re} p(z)>0$ in $U$.

A more general form of this lemma may be found in [2].

3. Main results. From the result of Hallenbeck and Ruscheweyh [1] (see also [2]) we find that if $P$ is analytic in $U$ with $P(0)=1$ then

$$
\operatorname{Re}\left[P(z)+z P^{\prime}(z)\right]>\beta \text { implies } P(z) \prec \beta+(1-\beta) l(z), z \in U,
$$

and

$$
\operatorname{Re}\left[P(z)+\frac{1}{2} z P^{\prime}(z)\right]>\beta \text { implies } P(z) \prec \beta+(1-\beta) L(z), z \in U,
$$

where $\prec$ stands for usual subordination, and $l$ and $L$ defined by $l(z)=$ $-1-(2 / z) \log (1-z)$ and $L(z)=2((l(z)-1) / z)-1$ are convex (univalent) in $U$. In view of the fact that the coefficients in the series expansions of $l$ and $L$ are positive, we easily deduce that $\operatorname{Re} l(z)>2 \ln 2-1$ and $\operatorname{Re} L(z)>3-4 \ln 2$ in $U$. Also it is easily seen that $l(U) \subset\{w \in \mathbb{C}:|\arg w|<\pi / 3\}$. Using a result of Mocanu et al. [4] we can replace $\pi / 3$ by $\theta$, where $\theta$ lies between 0.911621904 and 0.911621907 . This combined with the result of Robertson [7] yields that $l(U) \subset \Omega_{1} \cap \Omega_{2} \cap \Omega_{3}$ with $\Omega_{1}=\{w \in \mathbb{C}: \operatorname{Re} w>2 \ln 2-1\}$, $\Omega_{2}=\{w \in \mathbb{C}:|\arg w|<0.911621907\}$ and $\Omega_{3}=\{w \in \mathbb{C}:|\operatorname{Im} w|<\theta\}$.

THEOREM 1. If $\delta=(2 \ln 2-1)(3-2 \ln 2) /[3-(2 \ln 2-1)(3-2 \ln 2)]=$ $0.262 \ldots$ and $f \in R(-\delta)$ then the function $F$ defined by (1) is starlike in $U$.

Proof. From (1) we deduce

$$
z F^{\prime \prime}(z)+F^{\prime}(z)=f^{\prime}(z), \quad z \in U .
$$

Let $P(z)=F^{\prime}(z)$ and $Q(z)=F(z) / z$. Since $\operatorname{Re} f^{\prime}(z)>-\delta$ in $U$, by using (4) and (6) we find that $\operatorname{Re} F^{\prime}(z)>-\delta+(1+\delta)(2 \ln 2-1)$ for $z \in U$. Again by using (4) this in turn implies $\operatorname{Re}[Q(z)]>2 \delta>0, z \in U$.

Now if we set $p(z)=z F^{\prime}(z) / F(z)$ then $p$ is analytic in $U, p(0)=1$ and $f^{\prime}(z)=m\left(p(z), z p^{\prime}(z) ; z\right)$, where $m(u, v ; z)=\left(u^{2}+v\right) Q(z)$. To prove $F \in S^{*}$, it is enough to show $\operatorname{Re} p(z)>0$ in $U$. Since $\operatorname{Re} f^{\prime}(z)>-\delta$ in $U$, by (6), we get $\operatorname{Re} m\left(p(z), z p^{\prime}(z) ; z\right)>-\delta$ in $U$. Now for all real $x, y \leq-\left(1+x^{2}\right) / 2$ and $z \in U$, we have $\operatorname{Re} m(i x, y ; z)=\left(y-x^{2}\right) \operatorname{Re} Q(z) \leq-\left(1+3 x^{2}\right) \operatorname{Re}(Q(z) / 2) \leq$ $-\delta$ and so applying Lemma A with $\Omega=\{w \in \mathbb{C}: \operatorname{Re} w>-\delta\}$, we get $\operatorname{Re} p(z)>0$ in $U$. Hence the theorem. 
For the proof of Theorem 2 we prove the following lemmas. Theorem 2 further improves Theorem 1 .

LEMmA 1. If $g \in R(\beta)$ then $G$ defined by (2) belongs to $R(\beta+(1-\beta)(3-$ $4 \ln 2))(\beta<1)$.

Proof. From (2) we deduce

$$
\begin{aligned}
z G^{\prime}(z)+G(z) & =2 g(z), \\
z G^{\prime \prime}(z)+2 G^{\prime}(z) & =2 g^{\prime}(z) .
\end{aligned}
$$

Since $g \in R(\delta)$, by using (5), we obtain

$$
G^{\prime}(z) \prec \beta+(1-\beta) L(z), \quad z \in U,
$$

and so $\operatorname{Re} G^{\prime}(z)>\beta+(1-\beta)(3-4 \ln 2), z \in U$. Here $L(z)$ is as defined earlier. This proves Lemma 1 .

Lemma 2. Let $M=2(2 \ln 2-1)(1-\ln 2), \theta=0.911621907, N=\tan \theta$, $a=4(1+M)^{2}-\frac{4}{3} N^{2}(2 \ln 2-1)^{4}-4, b=-4(1-2 M)(1+M)-\frac{8}{3}(2 \ln 2-1)^{4} N^{2}$, $c=(1-2 M)^{2}-\frac{2}{3}(2 \ln 2-1)^{2} N$ and $\varrho=\left(-b-\left(b^{2}-4 a c\right)^{1 / 2}\right) /(2 a)$. Suppose that $Q$ is a complex function with $Q(0)=1$ satisfying

$$
\begin{gathered}
Q(U) \subset E_{1} \cap E_{2} \cap E_{3} \text { where } \\
E_{1}=\{w \in \mathbb{C}: \operatorname{Re} w>1-2 M(1+\varrho)\}, \\
E_{2}=\{w \in \mathbb{C}:|\arg (w-(1-2(2 \ln 2-1)(\varrho+1)))|<\theta\}, \\
E_{3}=\{w \in \mathbb{C}:|\operatorname{Im} w|<2(2 \ln 2-1)(\varrho+1) \pi\} .
\end{gathered}
$$

If $p$ is analytic in $U$ with $p(0)=1$ and if

$$
\operatorname{Re} Q(z)\left[z p^{\prime}(z)+p^{2}(z)+p(z)\right]>-2 \varrho, \quad z \in U,
$$

then $\operatorname{Re} p(z)>0$ in $U$.

Throughout the paper $M, \theta, N, a, b, c, \varrho$, and $E$ 's are all as defined above.

Proof of Lemma 2. If we let $m(u, v ; z)=Q(z)\left(v+u^{2}+u\right) / 2$ then for all $x, y \leq-\left(1+x^{2}\right) / 2$ and $z \in U$, we have

$$
\begin{aligned}
\operatorname{Re} m(i x, y ; z) & =\left[\left(y-x^{2}\right) \operatorname{Re} Q(z)-x \operatorname{Im} Q(z)\right] / 2 \\
& \leq-\left[3 x^{2} \operatorname{Re} Q(z)+2 x \operatorname{Im} Q(z)+\operatorname{Re} Q(z)\right] / 4 .
\end{aligned}
$$

Thus $\operatorname{Re} m(i x, y ; z) \leq-\varrho$ if $Q$ satisfies

$$
\left[(X-2 \varrho)^{2} /(2 \varrho)^{2}\right]-\left[Y^{2} /\left(3(2 \varrho)^{2}\right)\right] \geq 1
$$

where $X=\operatorname{Re} Q(z)$ and $Y=\operatorname{Im} Q(z)$. 
Since $Q$ satisfies (9), to prove (10) it is enough to show that the point $\left(X_{0}, Y_{0}\right)$ with $X_{0}=1-2 M(\varrho+1)$ and $Y_{0}=2(2 \ln 2-1)^{2}(\varrho+1) \tan \theta$ lies on the hyperbola $\left[(X-2 \varrho)^{2} /(2 \varrho)^{2}\right]-\left[Y^{2} /\left(3(2 \varrho)^{2}\right)\right]=1$. Thus by substituting this value in this hyperbola, we get, by a simple calculation,

$$
a \varrho^{2}+b \varrho+c=0 .
$$

Hence by hypothesis, we deduce that $\operatorname{Re} m(i x, y ; z) \leq-\varrho$ for all $z \in U$. Now by Lemma A, with $\Omega=\{w \in \mathbb{C}: \operatorname{Re} w>-\varrho\}$, we obtain $\operatorname{Re} p(z)>0$ in $U$. This completes the proof of Lemma 2 .

Remark 1 . If we let $\varrho^{\prime}$ and $\varrho^{\prime \prime}$ be the roots of the quadratic equation (12) then the approximate calculations show that

$$
\begin{aligned}
\varrho^{\prime} & =\left(-b-\left(b^{2}-4 a c\right)^{1 / 2}\right) /(2 a) \approx 0.09032572 \ldots, \\
\varrho^{\prime \prime} & =\left(-b+\left(b^{2}-4 a c\right)^{1 / 2}\right) /(2 a) \approx 1.2113303378 \ldots
\end{aligned}
$$

(Here $a \approx 2.071919132 \ldots, b \approx-2.701014071, c \approx 0.227066802 \ldots$ and $\left.b^{2}-4 a c=\frac{80}{3} \tan ^{2} \theta(2 \ln 2-1)^{4}+16(1-2 M)^{2} \approx[2.326718893 \ldots]^{2}.\right)$

Theorem 2. Let $\varrho$ be as defined in Lemma 2, i.e., $\varrho \approx 0.09032572 \ldots$ and $g \in R(-\varrho)$. Then the Libera transform $G$ defined by $(2)$ is in $S^{*}$.

Pr o of. Since $g \in R(-\varrho)$, by using Lemma 1 we obtain $G \in R(\beta)$ with

$$
\beta=-\varrho+(1+\varrho)(3-4 \ln 2)=1-2(2 \ln 2-1)(\varrho+1) .
$$

Now using (4) and the fact that $G \in R(\beta)$ we get

$$
(G(z) / z) \prec \beta+(1-\beta) l(z), \quad z \in U,
$$

where $l(z)=-1-(2 / z) \log (1-z)$. By (13), a simple calculation yields $\beta+(1-\beta)(2 \ln 2-1)=1-2 M(1+\varrho)$. This, from (14) and the observation made earlier, shows that the complex function $Q$ defined by $Q(z)=G(z) / z$ satisfies (9). If we set $p(z)=z G^{\prime}(z) / G(z)$, by using (2) we obtain

$$
z G^{\prime \prime}(z)+2 G^{\prime}(z)=2 g^{\prime}(z) .
$$

Since $\operatorname{Re} g^{\prime}(z)>-\varrho$ in $U$, by using (15) we easily get

$$
\operatorname{Re}\left\{Q(z)\left[z p^{\prime}(z)+p^{2}(z)+p(z)\right]\right\}>-2 \varrho, \quad z \in U,
$$

and by Lemma 2 we deduce $\operatorname{Re} p(z)>0$ in $U$, which shows that $G \in S^{*}$. Hence the theorem.

The following theorem can be proved along similar lines and so we omit its proof.

TheOREM 3. If $h \in A$ satisfies $\operatorname{Re}\left\{h^{\prime}(z) h(z) / z\right\}>-\varrho$ in $U$ then the function $H$ defined by $H(z)=\int_{0}^{z}(h(t) / t) d t$ is starlike in $U$.

Remark 2. In [6], the author showed that for $f \in A$ and $1 / 6 \leq \beta<1$, $\operatorname{Re}\left[h^{\prime}(z) h(z) / z\right]>\beta((3 \beta-1) / 2)$ implies $\operatorname{Re}(f(z) / z)>\beta$ in $U$. 
Remark 3. For $\alpha \geq 0$ and $\beta<1$, let $R(\alpha, \beta)$ be the class of functions $f$ in $A$ satisfying $\operatorname{Re}\left[f^{\prime}(z)+\alpha z f^{\prime \prime}(z)\right]>\beta$ for $z$ in $U$. From a result of Ponnusamy and Karunakaran [5], we have $R(\alpha, \beta) \subset R\left(\alpha^{\prime}, \beta+\left(\alpha-\alpha^{\prime}\right)(1-\right.$ $\beta) /(2+\alpha))$ for all $\alpha>\alpha^{\prime} \geq 0$. This relation and Theorem 2 show that

$$
R(\alpha,(-2 \varrho(2+\alpha)+1-2 \alpha) / 5) \subset S^{*} \quad \text { for all } \alpha \geq 1 / 2 .
$$

As an immediate consequence of the above observation, we have

ThEOREM 4. If $f \in A$ satisfies $\operatorname{Re}\left[f^{\prime}(z)\right]>(-2 \varrho(2+\alpha)+1-2 \alpha) / 5$, $z \in U$, for $\alpha \geq 1 / 2$, then the function $F$ defined by

$$
F(z)=\alpha z^{1-1 / \alpha} \int_{o}^{z} f(t) t^{1 / \alpha-2} d t
$$

is in $S^{*}$.

Corollary. If $f \in A$ satisfies $\operatorname{Re} f^{\prime}(z)>-(6 \varrho+1) / 5 \approx 0.3083908 \ldots$ for $z$ in $U$, then the function $F$ defined by (1) is starlike in $U$.

The above corollary improves Theorem 1.

Remark 4. For $g$ defined by $g(z)=z(2+z) / 2(1-z)$ (and hence $g$ satisfies $\operatorname{Re}\left[z g^{\prime}(z) / g(z)\right]>-1 / 2$ in $\left.U\right)$ it is well known that the corresponding Libera transform $G$ is starlike in $U$. On the other hand, a simple calculation shows that $g \in R(-1 / 8)$. Hence the natural problem which arises is to find the best possible $\varrho^{\prime}(>\varrho)$ such that $g \in R\left(-\varrho^{\prime}\right)$ implies $G$ is starlike in $U$.

I would like to thank Prof. Dr. K.-J. Wirths for encouraging me by sending a copy of [3].

\section{References}

[1] D. J. Hallenbeck and S. Ruscheweyh, Subordination by convex functions, Proc. Amer. Math. Soc. 52 (1975), 191-195.

[2] S. S. Miller and P. T. Mocanu, Differential subordination and univalent functions, Michigan Math. J. 28 (1981), 157-171.

[3] P. T. Mocanu, On starlikeness of Libera transform, Mathematica (Cluj) 28 (51) (1986), 153-155.

[4] P. T. Mocanu, D. Ripeanu and M. Popovici, Best bound for the argument of certain analytic functions with positive real part, preprint, "Babeş-Bolyai" Univ., Fac. Math., Res. Semin. 5 (1986), 91-98.

[5] S. Ponnusamy, On a subclass of $\lambda$-spirallike functions, Mathematica (Cluj), to appear.

[6] S. Ponnusamy and V. Karunakaran, Differential subordination and conformal mappings, Complex Variables Theory Appl. 11 (1989), 79-86.

[7] M. S. Robertson, An extremal problem for functions with positive real part, Michigan Math. J. 11 (1964), 327-335. 
[8] R. Singh and S. Singh, Starlikeness and convexity of certain integrals, Ann. Univ. Mariae Curie-Skłodowska Sect. A 35 (16) (1981), 145-148.

DEPARTMENT OF MATHEMATICS

INDIAN INSTITUTE OF TECHNOLOGY

KANPUR 208 016, INDIA

Reçu par la Rédaction le 5.3.1990 